\section{Domínios de atividade física e escolaridade em São Paulo, Brasil: estudo transversal seriado, 2003 e 2008}

\author{
Domains of physical activity and education in \\ São Paulo, Brazil: a serial cross-sectional \\ study in 2003 and 2008
}

\author{
Los dominios de actividad física y educación \\ en São Paulo: estudio transversal, serie \\ 2003 y 2008
}

\begin{abstract}
This study aimed to estimate the prevalence of physical activity in different domains and the association with schooling, using a serial crosssectional population-based design comparing data from two editions of a health survey in the city of São Paulo, Brazil. Participation included 1,667 adults in 2003 and 2,086 in 2008. Probabilistic sampling was performed by two-stage clus ters. The long version of International Physical Activity Questionnarie (IPAQ) allowed evaluating multiple domains of physical activity. Poisson regression was used. Men were more active in their leisure time and at work and women in the home. Schooling was associated directly with leisure-time activity (2003 and 2008) and inversely with work-related physical activity (2003) for men and for women in housework. The studies showed that Brazilians with less schooling are becoming less active, so that intervention strategies should consider different educational levels. Interventions in the urban space and transportation can increase the opportunities for physical activity and broaden access by the population.
\end{abstract}

Motor Activity; Health Care (Public Health); Educational Status; Health Surveys
Ana Paula de Oliveira Barbosa Nunes 1 Olinda do Carmo Luiz 1 Marilisa Berti Azevedo Barros 2 Chester Luis Galvão Cesar 3 Moisés Goldbaum 1

\section{Resumo}

O objetivo foi estimar a prevalência de atividade física em diferentes domínios e a sua associação com escolaridade. Trata-se de estudo seriado de base populacional de duas edições do Inquérito de Saúde de São Paulo, Brasil. Participaram 1.667 adultos, em 2003, e 2.086, em 2008. Amostragem probabilística foi realizada por conglomerados em dois estágios. O International Physical Activity Questionnaire (IPAQ) longo permitiu avaliar diversos domínios de atividade física. Foi utilizada regressão de Poisson. Os homens foram mais ativos no lazer e no trabalho; as mulheres, no lar. Encontrou-se aumento de ativos no deslocamento e na atividade total em homens e, também, no trabalho, entre as mulheres. A escolaridade foi associada de forma direta com lazer (2003 e 2008) e de forma inversa com o trabalho (2003), para os homens, e casa, para as mulheres; pessoas com menor nível de escolaridade estão se tornando menos ativas. As estratégias de intervenção devem considerar os níveis de escolaridade. Intervenções no espaço urbano e no transporte podem aumentar a oportunidade e o acesso mais amplo da população à atividade física.

Atividade Motora; Atenção à Saúde; Escolaridade; Inquéritos Epidemiológicos 


\section{Introdução}

Globalmente, as doenças crônicas não transmissíveis são responsáveis por aproximadamente dois terços das mortes 1; em 2007, responderam por $72 \%$ dos óbitos no Brasil 2. Dentre os seus principais fatores de risco, a inatividade física vem sendo reconhecida, na literatura científica, como importante comportamento modificável 3,4, sendo determinante em $3 \%$ a $5 \%$ das principais doenças crônicas e em 5,31\% das mortes por todas as causas em nosso país. Este percentual corresponde a 53.673 mortes em $2008{ }^{5}$.

Por outro lado, a atividade física é um importante fator de promoção da saúde, implicando diretamente a redução de mortalidade e/ou a prevalência e surgimento mais tardio das doenças crônicas não transmissíveis 6 , sendo considerada como determinante para longevidade 6,7,8,9. As recomendações da Organização Mundial da Saúde (OMS) para adultos são de, pelo menos, 150 minutos semanais de atividade física moderada 10. Contudo, estudos que analisaram os domínios de atividade física em separado (lazer, transporte, trabalho, doméstico) apresentaram resultados conflitantes: alguns mostraram ausência de efeito ${ }^{11}$, outros apontaram impactos importantes sobre a saúde da população 12 e há ainda aqueles que encontraram malefícios 13,14, dependendo do domínio analisado 11,12,13,14.

Apesar do conhecimento a respeito do efeito deletério da inatividade física na saúde, seus níveis estão aumentando em muitos países 15,16. Estima-se que $31,1 \%$ da população mundial adulta não alcançam as recomendações mínimas preconizadas pela OMS 10 , com os percentuais variando entre $17 \%$, no sudeste da Ásia, a 43,3\%, nas Américas e no Mediterrâneo Oriental 3.

Além do impacto da inatividade física sobre a carga de doenças crônicas, a desigualdade social vem ocupando lugar de destaque no cenário mundial como fator explicativo das condições de saúde das populações e dos seus agravos à saúde, sendo este aspecto complexo e multidimensional 17,18. A desigualdade de poder, renda, bens e serviços, além das circunstâncias de vida dessas pessoas, contribui para a má condição de saúde e taxas mais elevadas de mortalidade 17,18. Os gradientes socioeconômicos vêm sendo observados há algum tempo na relação com os níveis de atividade física 19. As desigualdades em saúde e os diferentes padrões de comportamento relacionados ao estilo de vida, como o tabagismo e a alimentação 20,21, levaram a um maior interesse em investigar as desigualdades relacionadas à atividade física 22

Os gradientes socioeconômicos vêm sendo investigados por intermédio da mensuração do nível de escolaridade, variável que tem relativa estabilidade na idade adulta, reflete adequadamente o nível socioeconômico da população e é de fácil recolha 23. Apesar de existirem alguns estudos que investigam a relação entre nível de escolaridade e inatividade física, poucos avaliam essa relação considerando os diferentes domínios da atividade física: lazer, casa, trabalho e transporte 24,25 . Os resultados dessas pesquisas mostram que indivíduos com mais escolaridade tendem a ser mais ativos no domínio lazer 24 e que, por outro lado, indivíduos com menos anos de estudo tendem a ser mais ativos no trabalho 25 .

Diante disso, o objetivo do trabalho é estimar a prevalência de atividade física em diferentes domínios entre homens e mulheres adultos residentes no Município de São Paulo, Brasil, nos anos de 2003 e 2008, explorando a influência do nível de escolaridade nos padrões de atividade física.

\section{Métodos}

Estudo transversal seriado, cujos dados foram retirados do Inquérito de Saúde de São Paulo (ISA-Capital) dos anos 2003 e 2008 26,27. O ISA-Capital é um inquérito periódico de base populacional, que se vale de amostra probabilística do Município de São Paulo, com o objetivo de avaliar condições de vida e situação de saúde, incluindo o uso de serviços de saúde públicos e privados. A população do estudo é constituída pelos moradores da área urbana do município, selecionados em amostras independentes nos dois anos de estudo.

A metodologia foi a mesma em 2003 e 2008. O plano de amostragem foi por conglomerado, realizado em dois estágios. No primeiro, foram sorteados sessenta setores censitários, subdivisão territorial definida pelo Instituto Brasileiro de Geografia e Estatística (IBGE) para operacionalizar o censo demográfico. Cada setor censitário é a menor unidade territorial adequada à realização da coleta de dados por um pesquisador. É formado por área contínua e constitui um conjunto de quadras.

De posse da relação de domicílios que integram os setores censitários sorteados, no segundo estágio foram sorteadas as residências onde seus moradores foram entrevistados. Para garantir a representatividade sociodemográfica, o processo de amostragem foi realizado de forma que a amostra tivesse a mesma distribuição percentual de sexo, idade e nível de escolaridade da população do município. Tendo por base a estimativa de uma prevalência de 50\%, com nível de 95\% de confiança, erro de amostragem de 0,06 e efeito de 
delineamento de 1,5, o tamanho da amostra foi definido em 3.360 entrevistas. Maiores detalhes sobre a amostragem podem ser acessados de forma completa em outras publicações 26,27.

Para o presente estudo, foram analisados os dados dos entrevistados com idade igual ou superior a vinte anos, de ambos os sexos, não institucionalizados.

A coleta de dados do ISA-Capital foi realizada por questionário estruturado em blocos temáticos (condições de vida, estilo de vida, estado de saúde, como acesso e utilização de serviços de saúde) e sua aplicação foi realizada por entrevistadores com, pelo menos, o ensino médio completo, após receberem treinamento específico. Para assegurar o controle de qualidade da coleta de informações, foi feita supervisão direta dos entrevistadores com replicação de $10 \%$ das entrevistas.

As informações sobre atividade física foram coletadas por meio do International Physical Activity Questionnaire (IPAQ) em sua versão longa em português validada no Brasil 28. A forma longa do IPAQ avalia os domínios de atividade física (lazer, transporte, em casa e no trabalho) e classifica as atividades de cada domínio em moderadas e vigorosas, conforme a unidade de medida equivalente metabólico da atividade, a qual traduz o gasto calórico da atividade mediante o número de medidade equivalente metabóloco das atividades. A definição da atividade física em cada domínio considerou a última semana antes da entrevista, contabilizando a duração em minutos, (considerando, no mínimo, dez minutos contínuos), e a frequência semanal. Atividade física total foi obtida pela soma dos minutos despendidos em cada um dos domínios. Frequência semanal e o tempo total diário nas atividades em cada um dos quatro domínios foram avaliados segundo as diretrizes internacionais do IPAQ 29 .

Os participantes foram categorizados em inativos (I), quando não realizaram atividade física na última semana; insuficientemente ativos (IA), quando a atividade física na última semana ficou entre 10 e 149 minutos; e ativos (A), quando a duração da atividade física atingiu ou superou os 150 minutos semanais.

Quanto às variáveis consideradas na análise descritiva, as variáveis sociodemográficas foram: sexo (masculino; feminino), idade (em anos), cor da pele autorreferida (branca, preta, parda; amarela ou indígena), situação conjugal (sem companheiro, quando respondia solteiro, separado, desquitado e viúvo; com companheiro, quando respondia casado ou em união conjugal estável), renda per capita familiar (em salários mínimos) e trabalho (não; sim). As variáveis de estilo de vida foram: tabagismo (fumante atual ou fumante até seis meses anteriores; não fumante), índice de massa corporal (IMC), calculado com dados de estatura e pesos referidos.

No presente estudo, a escolaridade foi medida em anos completos de estudo com aprovação, categorizados em 0-4 anos; 5-8 anos; 9-11anos e $\geq 12$ anos de estudo.

\section{Análise estatística}

Houve dupla digitação do banco de dados em programa Epi-data, sendo realizada validação e consistência. A estatística descritiva foi empregada para a estimativa de prevalências e intervalos de 95\% de confiança (IC95\%).

Foi utilizado o teste estatístico qui-quadrado para comparação da atividade física realizada em diferentes domínios, entre os anos e entre os sexos, bem como para as características sociodemográficas e de estilo de vida. $\mathrm{O}$ teste quiquadrado foi também utilizado para avaliar a associação entre escolaridade e os domínios de atividade, além de comparar o domínio de atividade física entre os anos, por nível de escolaridade e sexo. O teste de tendência linear foi usado na análise das variáveis categóricas ordinais. $\mathrm{Na}$ análise ajustada, foi utilizada a regressão de Poisson com variância robusta. O nível de significância adotado foi de $5 \%$.

Todas as análises foram realizadas com o programa Stata 12.0 (Stata Corp., College Station, Estados Unidos). Foram incorporados os fatores de ponderação da amostra, baseados no sexo e idade dos entrevistados e na fração amostral do setor censitário, a partir do percentual de chefes de família com nível universitário.

\section{Resultados}

Em 2003, o ISA-Capital, entrevistou 3.357 pessoas. Foram considerados elegíveis para a presente pesquisa 1.667 participantes. Na edição de 2008, foram entrevistadas 3.271 pessoas, das quais 2.086 eram elegíveis. Em 2003, 98\% forneceram informações sobre atividade física nos diferentes domínios; em 2008, o percentual foi de 99,7\%, o que permitiu a análise de 1.634 participantes em 2003 e 2.084 em 2008.

Na Tabela 1, observam-se diferenças nas características sociodemográficas e de estilo de vida entre 2003 e 2008, para ambos os sexos. Entre os participantes do sexo masculino, as mudanças ocorreram na escolaridade ( $\mathrm{p}<0,001)$, renda $\mathrm{per}$ capita $(\mathrm{p}=0,001)$ e raça $(\mathrm{p}=0,014)$; para as mulheres, além das mesmas mudanças observadas para o masculino (escolaridade, $\mathrm{p}<0,001$; renda per capita, $\mathrm{p}=0,002$; raça $\mathrm{p}=0,036$ ), observou-se, 
Tabela 1

Características sociodemográficas e estilo de vida segundo sexo, no Município de São Paulo, Brasil, 2003 e 2008.

\begin{tabular}{|c|c|c|c|c|c|c|}
\hline \multirow[t]{3}{*}{ Variáveis } & \multicolumn{2}{|c|}{ Masculino } & \multirow[t]{3}{*}{ Valor de p * } & \multicolumn{2}{|c|}{ Feminino } & \multirow[t]{3}{*}{ Valor de p * } \\
\hline & 2003 & 2008 & & 2003 & 2008 & \\
\hline & $\% \star \star \star ~$ & $\%$ ** & & $\%$ ** & $\% * \star$ & \\
\hline Idade (anos) & 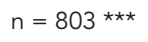 & $\mathrm{n}=848^{\star \star \star}$ & 0,211 & $\mathrm{n}=864 * \star \star$ & $\mathrm{n}=1.238 * \star \star$ & 0,063 \\
\hline $20-39$ & 53,3 & 49,6 & & 51,0 & 45,6 & \\
\hline $40-59$ & 32,6 & 36,2 & & 31,5 & 36,3 & \\
\hline $60-74$ & 10,4 & 9,5 & & 12,6 & 12,0 & \\
\hline$\geq 75$ & 3,7 & 4,8 & & 5,0 & 6,1 & \\
\hline Escolaridade (anos) & $\mathrm{n}=792 \star \star \star$ & $\mathrm{n}=848 \star \star \star$ & $<0,001$ & $\mathrm{n}=850 \star \star \star$ & $\mathrm{n}=1.238 \star \star \star$ & $<0,001$ \\
\hline $0-4$ & 27,2 & 16,7 & & 29,6 & 21,2 & \\
\hline $5-8$ & 19,2 & 18,5 & & 21,9 & 18,7 & \\
\hline $9-11$ & 23,8 & 34,7 & & 24,6 & 33,4 & \\
\hline$\geq 12$ & 29,9 & 30,2 & & 24,0 & 26,8 & \\
\hline Renda per capita (salários mínimos) & $\mathrm{n}=803^{* * *}$ & $\mathrm{n}=848^{\star \star \star}$ & 0,001 & $\mathrm{n}=864 * \star \star$ & $\mathrm{n}=1.238 \star \star \star$ & 0,002 \\
\hline$<0,5$ & 17,0 & 15,5 & & 22,0 & 18,4 & \\
\hline $0,5-1,0$ & 15,1 & 20,8 & & 24,7 & 20,3 & \\
\hline $1,0-2,5$ & 33,5 & 38,9 & & 26,8 & 36,2 & \\
\hline$>2,5$ & 34,3 & 24,8 & & 26,5 & 25,1 & \\
\hline Situação conjugal & $\mathrm{n}=791 \star \star \star$ & $\mathrm{n}=848^{\star \star \star}$ & 0,321 & $\mathrm{n}=848 * \star \star$ & $\mathrm{n}=1,238 * \star \star$ & 0,940 \\
\hline Sem parceiro & 37,4 & 34,4 & & 45,7 & 45,9 & \\
\hline Com parceiro & 62,7 & 65,7 & & 54,3 & 54,1 & \\
\hline Raça & $\mathrm{n}=784 * \star \star$ & 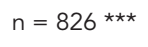 & 0,014 & $\mathrm{n}=845^{\star \star \star}$ & $\mathrm{n}=1.217 \star \star \star \star$ & 0,036 \\
\hline Branca & 67,3 & 61,2 & & 71,4 & 65,4 & \\
\hline Preta & 8,9 & 7,0 & & 7,5 & 7,8 & \\
\hline Parda & 23,8 & 31,8 & & 21,1 & 26,8 & \\
\hline Trabalho & $\mathrm{n}=776 * \star \star$ & $\mathrm{n}=848^{\star \star \star}$ & 0,247 & 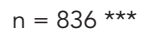 & $\mathrm{n}=1.238 * \star \star$ & $<0,001$ \\
\hline Não & 20,7 & 18,2 & & 49,2 & 39,3 & \\
\hline $\operatorname{Sim}$ & 79,3 & 81,8 & & 50,8 & 60,7 & \\
\hline Tabagismo & $\mathrm{n}=802 * \star \star$ & $\mathrm{n}=848 * \star \star$ & 0,861 & 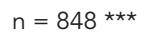 & $\mathrm{n}=1.238 * \star \star$ & 0,452 \\
\hline Não & 76,2 & 82,0 & & 75,7 & 80,3 & \\
\hline Sim & 23,9 & 18,0 & & 24,3 & 19,7 & \\
\hline IMC & 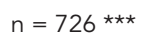 & $\mathrm{n}=837 \star \star \star$ & 0,072 & $\mathrm{n}=707 \star \star \star$ & $\mathrm{n}=1.193 \star \star \star \star$ & 0,096 \\
\hline Baixo peso & 5,6 & 3,2 & & 6,7 & 5,6 & \\
\hline Normal & 51,9 & 47,7 & & 56,7 & 52,6 & \\
\hline Sobrepeso & 30,1 & 37,7 & & 26,1 & 27,0 & \\
\hline Obesidade & 12,4 & 13,4 & & 10,5 & 14,8 & \\
\hline
\end{tabular}

* Valor de p teste qui-quadrado (diferença entre os anos);

** Porcentagens na amostra ponderada;

*** Número absoluto na amostra não ponderada.

ainda, aumento na proporção de trabalhadoras $(\mathrm{p}<0,001)$.

No período analisado, os resultados apontam, para o sexo masculino, aumento na proporção dos que realizam, pelo menos, 150 minutos de atividade física, com $\mathrm{p}=0,005$ (Tabela 2). Os homens apresentaram, também, diferença significativa no domínio transporte, entre os anos ( $\mathrm{p}<0,001$ ), com diminuição de inativos (quem não realiza atividade no transporte) e aumento expressivo na proporção de insuficientemente ativos (de 10 minutos a 149 minutos). Essa tendência não foi observada de forma significativa para o sexo feminino $(p=0,058)$; entre as mulheres, verificou-se aumento na atividade realizada no trabalho $(\mathrm{p}=0,049)$. As atividades em casa $\mathrm{e}$ no trabalho, entre homens, e no lazer e em casa, entre as mulheres, mantiveram-se estáveis. 
Tabela 2

Proporção de participantes segundo nível de atividade física em diferentes domínios. Município de São Paulo, Brasil, 2003 e 2008.

\begin{tabular}{|c|c|c|c|c|c|c|c|c|c|c|c|}
\hline \multirow[t]{3}{*}{ Domínios } & \multicolumn{4}{|c|}{2003} & \multicolumn{4}{|c|}{2008} & \multirow[t]{3}{*}{ Valor de $p$ ** } & \multicolumn{2}{|c|}{ Valor de $p$} \\
\hline & \multicolumn{4}{|c|}{$\%$ * } & \multicolumn{4}{|c|}{$\%$ * } & & \multirow[b]{2}{*}{2003} & \multirow[b]{2}{*}{2008} \\
\hline & $\mathrm{n} * \star \star$ & $I$ & IA & A & $\mathrm{n} * \star \star$ & I & IA & A & & & \\
\hline \multicolumn{12}{|l|}{ Masculino } \\
\hline Transporte & 779 & 42,1 & 26,8 & 31,1 & 846 & 29,4 & 43,3 & 27,4 & $<0,001$ & & \\
\hline Lazer & 779 & 53,4 & 17,7 & 29,0 & 846 & 52,6 & 16,5 & 30,9 & 0,790 & & \\
\hline Casa & 779 & 55,5 & 19,8 & 24,7 & 847 & 48,5 & 21,9 & 29,6 & 0,184 & & \\
\hline Trabalho & 779 & 53,2 & 5,9 & 40,9 & 843 & 50,5 & 5,9 & 43,6 & 0,748 & & \\
\hline Total & 779 & 10,2 & 11,0 & 78,9 & 842 & 4,6 & 11,9 & 83,5 & 0,005 & & \\
\hline \multicolumn{12}{|l|}{ Feminino } \\
\hline Transporte & 837 & 35,1 & 36,3 & 28,6 & 1233 & 28,8 & 43,6 & 27,7 & 0,074 & 0,016 & 0,969 \\
\hline Lazer & 837 & 72,7 & 12,3 & 15,1 & 1237 & 69,2 & 12,6 & 18,2 & 0,367 & $<0,001$ & $<0,001$ \\
\hline Casa & 837 & 22,3 & 17,2 & 60,5 & 1233 & 18,1 & 15,3 & 66,6 & 0,179 & $<0,001$ & $<0,001$ \\
\hline Trabalho & 837 & 73,6 & 3,8 & 22,7 & 1231 & 67,8 & 6,7 & 25,4 & 0,049 & $<0,001$ & $<0,001$ \\
\hline Total & 837 & 7,9 & 12,0 & 80,2 & 1224 & 5,7 & 8,6 & 85,7 & 0,058 & 0,496 & 0,118 \\
\hline
\end{tabular}

* Porcentagem de participantes na amostra ponderada por domínio e tempo de atividade física realizada, em minutos, no período de uma semana (minutos):

I = inativo (< 10 minutos ou nenhuma atividade); $\mid \mathrm{A}=$ insuficientemente ativo (de 10-149 minutos); $A=$ ativo ( $\geq 150$ minutos);

** Teste qui-quadrado;

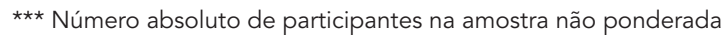

Em 2003, os homens foram mais ativos do que as mulheres no transporte $(\mathrm{p}=0,016)$.

Nos dois anos, houve maior proporção de homens que praticavam atividade física no lazer $(\mathrm{p}<0,001)$ e no trabalho $(\mathrm{p}<0,001)$, quando comparados com as mulheres. Por sua vez, estas faziam mais atividades domésticas $(\mathrm{p}<0,001)$ (Tabela 2).

Na Tabela 3, os resultados apontam diferença entre os anos 2003 e 2008 na atividade total (soma dos domínios) entre os mais escolarizados (E3), tanto para o sexo masculino ( $p=0,007)$, quanto para o feminino ( $\mathrm{p}=0,034)$. No lazer, entre os anos, observou-se diferença apenas para as mulheres de nível de escolaridade intermediário (E2, $p=0,043$ ). No trabalho, não se observou diferença entre os anos em ambos os sexos. Já para a atividade realizada em casa, diferença entre os anos foi observada para as mulheres com maior escolaridade $(E 3, p=0,005)$. No que se refere à atividade realizada no transporte, diferença entre os anos só foi observada para os homens com níveis de escolaridade E1 ( $p=0,043)$, E2 $(\mathrm{p}=0,014)$ e $\mathrm{E} 3(\mathrm{p}<0,001)$. Nas duas últimas colunas, as diferenças entre os sexos são observadas para todos os níveis de escolaridade.

Na Tabela 4, para o sexo masculino, resultado da análise ajustada mostra gradiente positivo entre atividade realizada no lazer e nível de escolaridade, observado tanto em 2003 (p de tendêcia =
0,005), quanto em 2008 (p de tendência < 0,001). Já para a atividade no trabalho, observa-se gradiente negativo em relação ao nível de escolaridade, o mesmo tendo sido observado em 2003 ( $p$ de tendência $=0,002$ ) e em 2008 ( $p$ de tendência = 032). Entretanto, apenas em 2003, os indivíduos com maior escolaridade (E3) apresentaram menor chance de serem ativos no trabalho $(\mathrm{RP}=0,5)$, o que não se repetiu em 2008, pelo aumento na proporção de ativos entre os mais escolarizados (E3). Na atividade em casa, não se observou gradiente na associação; somente em 2008, os indívíduos com cinco a oito anos de estudo apresentaram maior chance de serem ativos em casa $(R P=1,7)$, em comparação com os menos escolarizados (zero a quatro anos de estudo). Nas atividades realizadas no transporte e total (soma dos domínios), não se observou gradiente e/ou associação com nível de escolaridade.

Entre as mulheres, de forma semelhante à verificada para o sexo masculino, encontrouse o mesmo gradiente positivo entre atividade realizada no lazer e nível de escolaridade ( $\mathrm{p}$ de tendência $<0,001)$. O gradiente negativo relacionado com atividade realizada no trabalho em 2003 (p de tendência $=0,005$ ) não se repetiu em 2008 ( $\mathrm{p}$ de tendência $=0,168$ ), pela diminuição na proporção de ativas entre as menos escolarizadas. Atividade em casa apresenta gradiente negativo associado à escolaridade; em 2008, com 
Tabela 3

Proporção de participantes por nível de atividade física nos diferentes domínios e no total de atividade (soma dos domínios), segundo escolaridade, estratificado por sexo, no Município de São Paulo, Brasil, 2003 e 2008.

\begin{tabular}{|c|c|c|c|c|c|c|c|c|c|c|c|c|c|c|c|c|}
\hline \multirow[t]{3}{*}{ Domínios/Escolaridade * } & \multicolumn{6}{|c|}{ Masculino (\%) ** } & \multirow[t]{3}{*}{ Valor de $p^{\star \star \star}$} & \multicolumn{6}{|c|}{ Feminino $(\%) * \star$} & \multirow[t]{3}{*}{ Valor de $p \#$} & \multirow{2}{*}{\multicolumn{2}{|c|}{$\begin{array}{l}\text { Entre sexos } \\
\text { Valor de } p \text { * }\end{array}$}} \\
\hline & \multicolumn{3}{|c|}{2003} & \multicolumn{3}{|c|}{2008} & & \multicolumn{3}{|c|}{2003} & \multicolumn{3}{|c|}{2008} & & & \\
\hline & 1 & IA & A & $I$ & IA & A & & 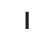 & IA & A & $I$ & IA & A & & 2003 & 2008 \\
\hline \multicolumn{17}{|l|}{ Lazer } \\
\hline EO & 73 & 9 & 18 & 67 & 17 & 15 & 0,075 & 81 & 10 & 9 & 77 & 12 & 11 & 0,562 & 0,031 & 0,774 \\
\hline E1 & 60 & 21 & 18 & 65 & 16 & 20 & 0,550 & 74 & 9 & 17 & 81 & 11 & 9 & 0,121 & 0,062 & 0,001 \\
\hline E2 & 48 & 13 & 39 & 53 & 15 & 31 & 0,341 & 76 & 15 & 9 & 74 & 9 & 16 & 0,043 & $<0,001$ & $<0,001$ \\
\hline E3 & 35 & 27 & 38 & 36 & 18 & 46 & 0,208 & 58 & 15 & 27 & 49 & 19 & 33 & 0,403 & 0,025 & 0,032 \\
\hline \multicolumn{17}{|l|}{ Trabalho } \\
\hline EO & 54 & 2 & 44 & 60 & 3 & 37 & 0,475 & 78 & 1 & 21 & 81 & 3 & 16 & 0,220 & $<0,001$ & $<0,001$ \\
\hline E1 & 42 & 4 & 54 & 38 & 6 & 56 & 0,689 & 69 & 4 & 27 & 62 & 3 & 35 & 0,370 & $<0,001$ & 0,001 \\
\hline E2 & 48 & 6 & 47 & 44 & 7 & 49 & 0,863 & 73 & 6 & 21 & 64 & 7 & 29 & 0,216 & $<0,001$ & $<0,001$ \\
\hline E3 & 65 & 11 & 25 & 60 & 7 & 34 & 0,175 & 73 & 4 & 23 & 66 & 11 & 22 & 0,139 & 0,118 & 0,069 \\
\hline \multicolumn{17}{|l|}{ Casa } \\
\hline EO & 53 & 23 & 24 & 54 & 24 & 23 & 0,959 & 20 & 11 & 69 & 19 & 15 & 66 & 0,587 & $<0,001$ & $<0,001$ \\
\hline E1 & 49 & 21 & 30 & 45 & 19 & 35 & 0,712 & 10 & 16 & 74 & 10 & 15 & 75 & 0,934 & $<0,001$ & $<0,001$ \\
\hline E2 & 53 & 21 & 26 & 46 & 21 & 32 & 0,487 & 17 & 19 & 64 & 11 & 17 & 72 & 0,292 & $<0,001$ & $<0,001$ \\
\hline E3 & 63 & 16 & 20 & 50 & 23 & 27 & 0,212 & 44 & 24 & 32 & 32 & 15 & 54 & 0,005 & 0,038 & 0,001 \\
\hline \multicolumn{17}{|l|}{ Transporte } \\
\hline EO & 41 & 30 & 29 & 37 & 34 & 29 & 0,697 & 41 & 34 & 24 & 34 & 46 & 20 & 0,065 & 0,406 & 0,024 \\
\hline E1 & 35 & 28 & 38 & 24 & 45 & 31 & 0,043 & 29 & 36 & 35 & 26 & 42 & 32 & 0,565 & 0,462 & 0,873 \\
\hline E2 & 43 & 25 & 33 & 32 & 41 & 27 & 0,014 & 26 & 41 & 33 & 24 & 46 & 30 & 0,600 & 0,011 & 0,168 \\
\hline E3 & 48 & 24 & 28 & 25 & 49 & 25 & $<0,001$ & 43 & 35 & 23 & 33 & 39 & 28 & 0,341 & 0,276 & 0,140 \\
\hline \multicolumn{17}{|l|}{ Total } \\
\hline EO & 15 & 13 & 73 & 12 & 16 & 72 & 0,714 & 12 & 9 & 79 & 10 & 11 & 79 & 0,559 & 0,440 & 0,183 \\
\hline E1 & 8 & 8 & 84 & 4 & 10 & 86 & 0,528 & 2 & 8 & 90 & 2 & 7 & 91 & 0,855 & 0,087 & 0,287 \\
\hline E2 & 6 & 9 & 85 & 4 & 10 & 86 & 0,587 & 3 & 13 & 84 & 3 & 7 & 90 & 0,185 & 0,432 & 0,361 \\
\hline E3 & 11 & 13 & 76 & 2 & 13 & 85 & 0,007 & 14 & 18 & 68 & 8 & 10 & 82 & 0,034 & 0,448 & 0,039 \\
\hline
\end{tabular}

* Valor de p: teste qui-quadrado (diferença entre os sexos por ano de estudo); E (escolaridade em anos completos de estudo): E0 = 0-4 anos; E1 = 5-8 anos;

E2 = 9-11 anos; E3 = $\geq 12$ anos;

** Porcentagem de participantes na amostra ponderada por domínio e tempo de atividade física realizada em minutos (minutos): I = inativo (< 10 minutos ou nenhuma atividade); $I A=$ insuficientemente ativo (de 10-149 minutos); $A=$ ativo ( $\geq 150$ minutos);

*** Valor de p: teste qui-quadrado (diferença entre os anos para o sexo masculino);

\# Valor de p: teste qui-quadrado (diferença entre os anos para o sexo feminino).

o aumento na proporção de ativas entre as mais escolarizadas, não houve associação. A atividade realizada no transporte não se mostrou associada à escolaridade em 2003; em 2008, com o aumento na proporção de ativos entre os mais escolarizados, estes passaram a ter maior chance de serem ativos no transporte.

A Tabela 5 apresenta a mudança de comportamento entre 2003 e 2008, em cada domínio, agora discriminados por intensidade/tipo de atividade realizada. Observa-se mudança significativa para o deslocamento feito a pé, tanto para o sexo masculino $(p<0,001)$, quanto para o fe- minino $(p=0,05)$. Para o sexo feminino, verificase ainda: diminuição no transporte de bicicleta ( $p=0,025)$; aumento na atividade moderada no lazer ( $p=0,014)$; aumento na atividade moderada realizada dentro de casa $(\mathrm{p}=0,047)$; aumento na caminhada realizada no trabalho $(\mathrm{p}=0,043)$. Nas quatro últimas colunas, estão os percentuais de mudança observados entre os anos de estudo, por nível de escolaridade. Redução na proporção de homens com menor escolaridade (E0) é observada para atividade vigorosa $(7 \%)$, moderada $(\downarrow 4,5 \%)$ e caminhada realizada no trabalho ( $16 \%)$, além de redução de $9 \%$ na atividade vigorosa re- 
Análise ajustada para atividade física nos domínios lazer, trabalho, casa, transporte e na soma deles (atividade total), em homens e mulheres, segundo escolaridade, no Município de São Paulo, Brasil, 2003 e 2008.

\begin{tabular}{|c|c|c|c|c|}
\hline \multirow{2}{*}{$\begin{array}{l}\text { Domínios/ } \\
\text { Escolaridade (anos) }\end{array}$} & \multicolumn{2}{|c|}{ Masculino } & \multicolumn{2}{|c|}{ Feminino } \\
\hline & 2003 & 2008 & 2003 & 2008 \\
\hline & Ajustada * & Ajustada * & Ajustada * & Ajustada * \\
\hline & RP (IC95\%) & RP (IC95\%) & RP (IC95\%) & RP (IC95\%) \\
\hline & $\mathrm{n}=705 * \star$ & $\mathrm{n}=797 * \star$ & $\mathrm{n}=775 * \star$ & $\mathrm{n}=1.171$ ** \\
\hline Lazer & 0,005 *** & $<0,001 * \star \star$ & $0,018 * \star \star$ & $<0,001 * \star \star$ \\
\hline $0-4$ & 1,00 & 1,00 & 1,00 & 1,00 \\
\hline $5-8$ & $1,0(0,5-1,8)$ & $1,2(0,6-2,1)$ & $2,6(1,3-5,4)$ & $0,9(0,5-1,6)$ \\
\hline $9-11$ & $2,0(1,3-3,1)$ & $1,8(1,1-3,0)$ & $1,4(0,6-3,1)$ & $1,7(1,0-2,9)$ \\
\hline \multirow[t]{2}{*}{$\geq 12$} & $1,7(1,0-2,8)$ & $2,5(1,5-4,3)$ & $3,6(1,9-6,9)$ & $3,1(1,8-5,1)$ \\
\hline & $\mathrm{n}=705 * \star$ & $\mathrm{n}=793 * \star$ & $\mathrm{n}=775 * \star$ & $\mathrm{n}=1.165^{* *}$ \\
\hline Trabalho & 0,002 *** & $0,032 * \star \star *$ & $0,005 * \star \star$ & 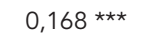 \\
\hline $0-4$ & 1,00 & 1,00 & 1,00 & 1,00 \\
\hline $5-8$ & $1,0(0,8-1,3)$ & $1,3(1,0-1,7)$ & $0,8(0,5-1,4)$ & $1,3(0,9-1,8)$ \\
\hline $9-11$ & $0,8(0,6-1,1)$ & $1,2(0,9-1,6)$ & $0,6(0,4-0,9)$ & $1,0(0,7-1,5)$ \\
\hline \multirow[t]{2}{*}{$\geq 12$} & $0,5(0,3-0,8)$ & $0,8(0,6-1,1)$ & $0,5(0,3-0,9)$ & $0,9(0,5-1,6)$ \\
\hline & $\mathrm{n}=705^{\star \star *}$ & $\mathrm{n}=797$ ** & $\mathrm{n}=775 * \star$ & $\mathrm{n}=1.167^{\star *}$ \\
\hline Casa & 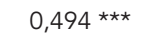 & $0,715 * \star *$ & $<0,001 * \star \star$ & 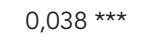 \\
\hline $5-8$ & 1,00 & 1,00 & 1,00 & 1,00 \\
\hline $9-11$ & $1,1(0,7-1,8)$ & $1,7(1,1-2,4)$ & $1,0(0,8-1,1)$ & $1,0(0,9-1,2)$ \\
\hline$\geq 12$ & $1,0(0,7-1,6)$ & $1,5(1,0-2,4)$ & $0,9(0,7-1,0)$ & $1,0(0,9-1,2)$ \\
\hline \multirow[t]{2}{*}{$5-8$} & $0,8(0,5-1,4)$ & $1,4(0,8-2,3)$ & $0,5(0,3-0,7)$ & $0,9(0,7-1,0)$ \\
\hline & $\mathrm{n}=704$ ** & $\mathrm{n}=705 * \star$ & $\mathrm{n}=775^{\star \star *}$ & $\mathrm{n}=1.167$ ** \\
\hline Transporte & $0,545 b$ & $0,204 b$ & $0,825 b$ & $0,055 b$ \\
\hline $0-4$ & 1,00 & 1,00 & 1,00 & 1,00 \\
\hline $5-8$ & $1,1(0,8-1,7)$ & $1,1(0,8-1,5)$ & $1,2(0,8-2,0)$ & $1,4(1,0-2,0)$ \\
\hline $9-11$ & $0,9(0,6-1,4)$ & $0,8(0,6-1,2)$ & $1,2(0,8-1,7)$ & $1,4(1,0-2,1)$ \\
\hline \multirow[t]{2}{*}{$\geq 12$} & $0,9(0,6-1,4)$ & $0,9(0,6-1,3)$ & $0,9(0,5-1,7)$ & $1,6(1,2-2,4)$ \\
\hline & $\mathrm{n}=705 * \star$ & $\mathrm{n}=705 * \star$ & $\mathrm{n}=775^{\star \star}$ & $\mathrm{n}=1.158$ ** \\
\hline Total & $0,961 * \star \star$ & $0,188 * \star \star$ & $0,029 * \star \star$ & $0,171 * \star \star$ \\
\hline $0-4$ & 1,00 & 1,00 & 1,00 & 1,00 \\
\hline $5-8$ & $1,0(0,9-1,2)$ & $1,1(1,0-1,2)$ & $1,1(1,0-1,2)$ & $1,0(1,0-1,1)$ \\
\hline $9-11$ & $1,0(0,9-1,2)$ & $1,1(1,0-1,2)$ & $1,0(0,9-1,1)$ & $1,0(1,0-1,1)$ \\
\hline$\geq 12$ & $1,0(0,9-1,1)$ & $1,1(1,0-1,3)$ & $0,9(0,7-1,0)$ & $1,0(0,9-1,1)$ \\
\hline
\end{tabular}

IC95\%: intervalo de 95\% de confiança; RP: razão de prevalência

* Modelo ajustado pelas variáveis associadas à escolaridade no modelo final, por sexo (masculino: renda, estado conjugal, idade, doença circulatória, saúde mental e raça; feminino: renda, raça, trabalho, idade, doença circulatória e saúde mental); ** número absoluto de participantes na amostra não ponderada;

*** Valor de $p$ de tendência.

alizada no lazer. Maior proporção de mulheres deixou de fazer atividade vigorosa $(51,6 \%)$, moderada $(27,1 \%)$ e caminhada no trabalho $(16 \%)$; redução de $19 \%$ é observada no quintal vigoroso.

\section{Discussão}

A análise de tendência do presente estudo apontou aumento na proporção de pessoas ativas. Quando considerados os domínios separadamente, observou-se aumento na proporção de insuficientemente ativos no transporte, ou seja, há maior proporção de pessoas fazendo atividade física nessa modalidade, porém em tempo in- 
Proporção de participantes que realizam mais de dez minutos por semana de algum tipo de atividade física, nos diferentes domínios, estratificados por tipo e intensidade de atividade, segundo grau de escolaridade e sexo, no Município de São Paulo, Brasil, 2003 e 2008.

\begin{tabular}{|c|c|c|c|c|c|c|c|c|c|c|c|c|c|c|c|c|}
\hline \multirow[t]{2}{*}{ Domínio } & \multicolumn{2}{|c|}{$\%$ * } & \multirow[t]{2}{*}{$\Delta \%$ ** } & \multirow{2}{*}{$\begin{array}{c}\text { Valor de } \\
p^{\star \star \star}\end{array}$} & \multicolumn{4}{|c|}{2003} & \multicolumn{4}{|c|}{2008} & \multicolumn{4}{|c|}{ 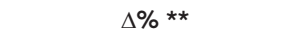 } \\
\hline & 2003 & 2008 & & & E1 \# & E2 \# & E3 \# & E4 \# & E1 \# & E2 \# & E3 \# & E4 \# & E1 & E2 & E3 & E4 \\
\hline \multicolumn{17}{|l|}{ Masculino } \\
\hline \multicolumn{17}{|l|}{ Transporte } \\
\hline Bicicleta & 6 & 4 & -32 & 0,168 & 4 & 10 & 6 & 6 & 3 & 11 & 3 & 2 & -30 & 9 & -55 & -58 \\
\hline A pé & 58 & 71 & 22 & $<0,001$ & 59 & 66 & 57 & 52 & 63 & 76 & 68 & 75 & 6 & 15 & 18 & 45 \\
\hline \multicolumn{17}{|l|}{ Lazer } \\
\hline Vigoroso & 13 & 17 & 29 & 0,136 & 4 & 8 & 19 & 21 & 3 & 6 & 19 & 28 & -13 & -20 & 0 & 37 \\
\hline Moderado & 22 & 19 & -14 & 0,363 & 4 & 22 & 30 & 32 & 8 & 15 & 21 & 25 & 97 & -33 & -28 & -21 \\
\hline Caminhada & 27 & 29 & 8 & 0,538 & 23 & 20 & 22 & 40 & 24 & 26 & 26 & 37 & 3 & 28 & 21 & -6 \\
\hline \multicolumn{17}{|l|}{ Casa } \\
\hline Q vigoroso & 17 & 20 & 14 & 0,481 & 20 & 20 & 13 & 17 & 18 & 20 & 24 & 16 & -9 & 2 & 79 & -8 \\
\hline Q moderado & 20 & 23 & 17 & 0,273 & 18 & 28 & 15 & 20 & 19 & 26 & 23 & 23 & 6 & -6 & 55 & 18 \\
\hline $\mathrm{C}$ moderado & 33 & 41 & 22 & 0,054 & 31 & 36 & 41 & 27 & 32 & 45 & 43 & 40 & 4 & 26 & 5 & 44 \\
\hline \multicolumn{17}{|l|}{ Trabalho } \\
\hline Vigoroso & 16 & 21 & 29 & 0,105 & 21 & 20 & 18 & 9 & 20 & 29 & 27 & 11 & -7 & 43 & 52 & 21 \\
\hline Moderado & 25 & 27 & 10 & 0,453 & 26 & 30 & 32 & 14 & 25 & 42 & 29 & 18 & -4 & 37 & -9 & 30 \\
\hline Caminhada & 37 & 36 & -3 & 0,819 & 35 & 43 & 42 & 32 & 29 & 45 & 41 & 29 & -16 & 4 & -2 & -10 \\
\hline \multicolumn{17}{|l|}{ Feminino } \\
\hline \multicolumn{17}{|l|}{ Transporte } \\
\hline Bicicleta & 3 & 1 & -69 & 0,025 & 1 & 6 & 2 & 2 & 0 & 0 & 2 & 0 & -100 & -100 & -1 & -76 \\
\hline A pé & 65 & 71 & 10 & 0,050 & 59 & 71 & 74 & 57 & 66 & 74 & 76 & 67 & 13 & 5 & 3 & 17 \\
\hline \multicolumn{17}{|l|}{ Lazer } \\
\hline Vigoroso & 5 & 7 & 50 & 0,140 & 2 & 5 & 2 & 12 & 2 & 3 & 6 & 16 & 16 & -31 & 193 & 31 \\
\hline Moderado & 5 & 9 & 89 & 0,014 & 3 & 4 & 7 & 6 & 5 & 6 & 8 & 15 & 91 & 42 & 20 & 154 \\
\hline Caminhada & 21 & 23 & 9 & 0,471 & 16 & 23 & 19 & 29 & 20 & 14 & 18 & 39 & 19 & -39 & -4 & 35 \\
\hline \multicolumn{17}{|l|}{ Casa } \\
\hline Q vigoroso & 32 & 30 & -8 & 0,496 & 36 & 38 & 40 & 13 & 29 & 34 & 36 & 20 & -19 & -12 & -11 & 52 \\
\hline Q moderado & 39 & 37 & -3 & 0,774 & 44 & 47 & 39 & 21 & 40 & 44 & 42 & 25 & -8 & -7 & 7 & 18 \\
\hline C moderado & 72 & 78 & 8 & 0,047 & 74 & 86 & 78 & 51 & 78 & 86 & 84 & 67 & 4 & 0 & 7 & 31 \\
\hline \multicolumn{17}{|l|}{ Trabalho } \\
\hline Vigoroso & 10 & 9 & -9 & 0,671 & 11 & 10 & 12 & 6 & 6 & 17 & 10 & 6 & -52 & 74 & -12 & -14 \\
\hline Moderado & 18 & 18 & 3 & 0,824 & 17 & 21 & 19 & 13 & 13 & 28 & 18 & 15 & -27 & 36 & -2 & 16 \\
\hline Caminhada & 18 & 24 & 35 & 0,043 & 13 & 17 & 19 & 24 & 11 & 26 & 29 & 28 & -18 & 55 & 51 & 19 \\
\hline
\end{tabular}

C moderado: atividade moderada dentro de casa; em negrito = diferença entre os anos por nível de escolaridade e tipo/intensidade/domínio de atividade realizada ( $<0,05)$; $\mathrm{Q}$ moderado: atividade moderada no quintal; $\mathrm{Q}$ vigoroso: atividade vigorosa no quintal.

* Proporção de pessoas por domínio e intensidade de atividade realizada;

** Delta \% de mudança entre 2003 e 2008;

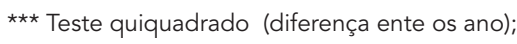

\# Porcentagem de participantes na amostra ponderada que realizam mais de 10 minutos por semana dos tipos e/ou intensidades de atividade física descritos nos diferentes domínios, segundo nível de escolaridade por número de anos de estudos em ordem crescente (E1 = 0-4 anos; E2 = 5-8 anos; E3 = 9-11 anos e E4 $=\geq 12$ anos no domínio casa.

suficiente, segundo as recomendações da OMS. O quadro observado no transporte ocorreu em virtude de um aumento de $21,7 \%$ no deslocamento a pé, entre homens, e 10,9\%, entre mulheres (Tabela 5). A OMS reconhece a atividade no transporte como um dos dez determinantes de saúde 30, pela grande contribuição que o transporte ativo diário pode ter na inserção da atividade física no cotidiano das pessoas. O transporte ativo minimiza a inatividade decorrente da falta de dinheiro e tempo ${ }^{31}$. Ademais, existe relação inversa entre caminhada e risco para doenças 
cardiovasculares e todas as causas de mortalidade, relação esta similar entre os gêneros 32 .

Percentual importante de pessoas com baixa escolaridade deixou de fazer atividade física, principalmente no trabalho. Considerando que as pessoas com baixa escolaridade já realizam menos atividade no lazer e apresentaram menor mudança na atividade física no transporte entre os anos, elas estão, consequentemente, mais vulneráveis aos efeitos deletérios da inatividade física ${ }^{33}$. Aqui, cabe acrescentar que, entre os anos, houve aumento na proporção de mulheres que trabalham e que realizam atividade física no trabalho. Todavia, fica claro que este aumento corresponde às categorias de maior escolaridade, uma vez que, entre as mulheres menos escolarizadas, foi observada queda na proporção de ativas no trabalho. As mudanças observadas harmonizam-se com o desenvolvimento econômico, que afeta a natureza do trabalho e influencia o comportamento, levando a uma mudança no gasto energético, com a redução da atividade física no trabalho e no deslocamento 34 .

Observamos aumento de escolaridade na população em foco entre os dois anos estudados, tendência populacional já apontada nos Censos Demográficos de 2000 e 2010. Esse fenômeno, no entanto, não interfere em nossos resultados, uma vez que trabalhamos com proporção.

No Brasil, nossa pesquisa pode ser comparada a três outros estudos: o primeiro foi realizado na cidade de Pelotas, Rio Grande do Sul, Brasil 35, o segundo, no Estado de São Paulo 36, e o terceiro baseou-se nos dados do Sistema de Vigilância de Fatores de Risco e Proteção para Doenças Crônicas por Inquérito Telefônico - VIGITEL (2006-2009), o qual analisou informações da população das capitais de estado e do Distrito Federal 37. Os dados de Pelotas 35 , que consideraram os quatro domínios em conjunto, mostraram diminuição no percentual de indivíduos ativos (de 59\% para $48 \%$, entre 2002 e 2007), resultado diferente do encontrado na presente investigação. Por outro lado, o estudo da população do Estado de São Paulo 36 mostrou prevalência mais alta de indivíduos ativos e aumento na prevalência entre 2002 e 2008, corroborando os resultados da nossa pesquisa. A diferença do resultado do estudo de Pelotas 35, comparado ao trabalho desenvolvido no Estado de São Paulo 36 e à presente investigação, poderia ser justificada pelos diferentes contextos populacionais e, em parte, pela ausência de programa para incentivar a prática de atividade física em Pelotas 38. Já os dados do estudo VIGITEL são apresentados em diferentes domínios e indicaram aumento na proporção de indivíduos ativos, especialmente no transporte, semelhante aos nossos achados. Na atividade em casa, foi ob- servada redução na proporção de mulheres ativas; nos demais domínios, não foram observadas diferenças significativas 37 .

A literatura internacional sobre tendências temporais de atividade física é, em grande parte, exclusiva de países de alta renda, cujos resultados apresentam diminuição dos níveis de atividade física realizada no trabalho 39 e aumento de atividade física no lazer 40. Resultado de estudo longitudinal realizado em Barcelona, Espanha (1994-2002), indica que 74,4\% dos indivíduos que eram considerados ativos no trabalho em 1994 não trocaram seus hábitos em 2002, enquanto $64,3 \%$ dos participantes que tinham hábitos sedentários em 1994 passaram a ser ativos nas atividades de lazer em 200240.

Divergências nas proporções de pessoas ativas e na tendência do comportamento ao longo do tempo são observadas em todo o mundo. Urbanização e fatores sociodemográficos, como raça, escolaridade, idade e aspectos étnicos, vêm sendo relatados na literatura como determinantes da atividade física 22,24,25. Alguns estudos apontam que diferenças nos níveis de atividade física no trabalho e doméstica mostraramse relacionadas com o grau de escolaridade das pessoas 41 .

O resultado das nossas análises indicou que, após período de cinco anos, indivíduos da categoria de maior escolaridade apresentaram aumento da atividade fisica total em ambos os sexos. Já os menos escolarizados, apesar de não apresentarem diferenças significativas entre os anos nos diferentes domínios, mostraram-se menos ativos na maior parte dos domínios, comparados aos indivíduos mais escolarizados.

O presente estudo indica associação positiva entre escolaridade e atividade física no lazer para ambos os sexos (2003 e 2008) e associação negativa entre atividade física doméstica e escolaridade para mulheres. Esses resultados também foram encontrados em pesquisas conduzidas em Pelotas, no sul do Brasil 41, na Colômbia 42 e na Itália 19. Quanto à atividade física realizada no trabalho, observa-se, ainda, associação negativa com escolaridade em homens e mulheres, porém apenas em 2003. Esses achados corroboram, em parte, o estudo realizado nos Estados Unidos, somente para o sexo masculino 43 , e o estudo realizado na China 39. Contudo, contradizem os achados do estudo realizado na zona leste de São Paulo 44, Ermelino Matarazzo, região onde predomina menor nível socieconômico. A pesquisa paulista utilizou critérios mais rígidos para classificar indivíduos como ativos no trabalho, o que pode ter levado a uma menor prevalência nessa categoria 44 . Outro estudo de tendência, realizado em Madri, entre 1995 e 2008, apresentou 
redução de atividade no trabalho de moderada intensidade entre os menos escolarizados 45 .

Quanto à prática de atividade física no transporte, estudo realizado com amostra representativa de croatas 46 e de iranianos 47 não encontrou associação significativa com escolaridade. Em contrapartida, em outros trabalhos, foram verificados níveis mais elevados de participação em transporte ativo em grupos populacionais ou regiões socialmente desfavorecidas 44 . Tal achado se deve à possibilidade de indivíduos com maiores níveis de escolaridade terem melhores níveis socieconômicos, permitindo aquisição de veículos motorizados e, portanto, acesso a outra opção de deslocamento. Já estudo realizado com empregados finlandeses apresentou redução na proporção de ativos no deslocamento em todos os níveis de escolaridade, entre 1978 e 200248.

A queda na proporção de pessoas ativas na categoria de menor escolaridade é, em grande parte, explicada por uma queda mais acentuada na atividade física realizada no trabalho e no trabalho doméstico, já que, para o lazer, os níveis de atividade são menores nesta população ${ }^{49}$. Estudo realizado no Brasil, com amostra representativa de Pelotas, também indica maior chance de ser inativo entre os menos escolarizados, nos dois momentos considerados (2002-2007) 35. Já na literatura internacional, estudo de tendência realizado no México 50 e estudo transversal em Bogotá, na Colômbia, não mostraram diferença na chance de ser ativo entre os diferentes níveis de escolaridade 42 .

Nos países em desenvolvimento, é necessário estimular um estilo de vida mais ativo nos estratos sociais menos favorecidos. Programas e políticas de incentivo à prática de atividade física devem considerar as diferenças socioeconômicas da população. Intervenções em ambientes públicos, as quais ofereçam instalações de fácil acesso e orientação adequada, modificam a escolha das pessoas em relação à atividade física 51 . A redução de barreiras físicas, psicológicas e financeiras associadas à atividade física no lazer poderia ser obtida com a maior oferta de espaços públicos adequados 51,52.

Maior adesão pode ser alcançada com a instalação de programas em praças e parques, ou mesmo ao longo das avenidas, em locais próximos às residências 53. Por outro lado, a percepção de pouca segurança, decorrente da violência urbana, e ambientes escuros podem constituir uma barreira importante à prática de atividade física nesses espaços 52 .

Já o deslocamento ativo (transporte) pode ser um domínio importante para alcançar os níveis recomendáveis de atividade física, sendo de fácil acesso, não implicando custos elevados e não adicionando demasiado tempo às atividades cotidianas. É uma boa forma de praticar atividade física sem grande alteração da rotina diária. Entretanto, as principais barreiras modificáveis relacionadas com a cidade (distância entre o ponto de saída e o destino, perigo relacionado com tráfego e segurança) e com o trabalho (local adequado e seguro para receber bicicletas, vestiários com chuveiros) devem ser objeto de intervenções, estimulando, assim, o envolvimento das pessoas no deslocamento ativo 54,55.

Do ponto de vista das limitações deste estudo, quanto ao uso de informação referida, destaca-se a mensuração da atividade física por meio de questionário, e não por método direto com o uso de acelerômetro, e o IMC calculado com base nas informações de peso e altura autoreportados. Pesquisas mostram que a informação referida pode superestimar a prevalência de fisicamente ativos, por se tratar de um comportamento socialmente desejável 56. Quanto ao IMC referido, este se mostrou válido para ser utilizado na classificação antropométrica populacional, com obtenção de sensibilidade e especificidade relativamente altas 57 .

Embora nem todos os estudos apresentem concordância entre os escores contínuos oriundos de questionários e de acelerômetro ${ }^{58,59}$, a categorização dos participantes em grupos de atividade física apresenta concordância de moderada a elevada 60 , o que justifica a utilização de questionários 61,62. O uso do mesmo instrumento nas duas avaliações é um ponto forte pelo alto grau de comparabilidade metodológica.

A presente pesquisa aponta importantes aspectos da prática de atividade física, no entanto um período maior ainda precisa ser estudado para consolidar os achados. Apesar disso, os dados permitem uma sinalização para algumas medidas de intervenção, buscando potencializar a tendência de aumento do nível da atividade física. A identificação de subgrupos mais vulneráveis, em especial os menos escolarizados, vai exigir ampla gama de estratégias e a criação de infraestrutura de apoio, com a proposta de interromper o declínio da atividade física para esta população. Intervenções no espaço urbano, nos locais de trabalho e no transporte podem aumentar a oportunidade e o acesso mais amplo à atividade física. Torna-se necessário o investimento em políticas que incentivem essa prática em todos os domínios, por meio da ação intersetorial, envolvendo prioritariamente as áreas de saúde, educação, esporte, meio ambiente, segurança, transporte e comunicação social, garantindo-se a participação da sociedade na definição do escopo e desenho dessas políticas. 


\section{Resumen}

El objetivo fue estimar la prevalencia de actividad física en diversos campos y su asociación con la escolaridad. Se realizó una serie basada en la población de dos ediciones de la Encuesta Municipal de Salud de São Paulo, Brasil. 1.667 adultos en 2003 y 2.086 participaron en 2008. Se realizó un muestreo probabilístico por conglomerados en dos etapas. El International Physical Activity Questionnarie (IPAQ) largo permite evaluar múltiples dominios de actividad física. Se utilizó la regresión de Poisson. Los hombres estaban más activos en el ocio y en el trabajo, y las mujeres en casa. El incremento de activos en desplazamiento y actividad total en los hombres y, también en el trabajo, entre las mujeres. La escolarización estaba directamente asociada con el ocio (2003 y 2008), y de forma inversa, con el trabajo (2003) para hombres y mujeres, además, las personas con menor nivel de educación son cada vez menos activas. Las estrategias de intervención deben considerar los niveles de escolaridad. Las intervenciones en el espacio urbano y en el transporte pueden aumentar la oportunidad y el acceso más amplio de la población a la actividad física.

Actividad Motora; Atención a la Salud; Escolaridad; Encuestas Epidemiológicas

\section{Colaboradores}

A. P. O. B. Nunes realizou a proposta do artigo, a revisão da literatura, a análise dos dados e a redação do artigo. O. C. Luiz contribuiu na orientação do trabalho, na interpretação dos dados e na redação do artigo. M. B. A. Barros, C. L. G. Cesar e M. Goldbaum contribuiram na revisão do artigo e aprovação da versão final do artigo

\section{Agradecimentos}

À Coordenação de Aperfeiçoamento de Ensino de Pessoal de Nível Superior (CAPES), pela bolsa de doutorado, e à Secretaria Municipal de Saúde de São Paulo (SMS-SP), pelo financiamento do trabalho de campo.

\section{Referências}

1. World Health Organization. Global status report on noncommunicable diseases 2010. Geneva: World Health Organization; 2011.

2. Schimidt MI, Duncan BB, Silva GA, Menezes AM, Monteiro CA, Barreto SM, et al. Doenças crônicas não transmissíveis no Brasil: carga e desafios atuais. Lancet 2011:61-74.

3. Hallal PC, Andersen LB, Bull FC, Guthold R, Haskell W, Ekelund U, et al. Global physical activity levels: surveillance progress, pitfalls, and prospects. Lancet 2012; 380:247-57.

4. Lim SS, Vos T, Flaxman AD, Danaei G, Shibuya K, Adair-Rohani $\mathrm{H}$, et al. A comparative risk assessment of burden of disease and injury attributable to 67 risk factors and risk factor clusters in 21 regions, 1990-2010: a systematic analysis for the Global Burden of Disease Study 2010. Lancet 2012; 380:2224-60.
5. de Rezende LF, Rabacow FM, Viscondi J YK, Luiz OC, Matsudo VK, Lee IM. Effect of Physical Inactivity on Major Non-Communicable Diseases and Life Expectancy in Brazil. J Phys Act Health 2015; 12:299-306.

6. Myers J, Kaykha A, George S, Abella J, Zaheer N, Lear S, et al. Fitness versus physical activity patterns in predicting mortality in men. Am J Med 2004; 117:912-8.

7. Wen CP, Wai JPM, Tsai MK, Yang YC, Cheng TY, Lee MC, et al. Minimum amount of physical activity for reduced mortality and extended life expectancy: a prospective cohort study. Lancet 2011; 378:1244-53.

8. World Health Organization. World health day 2002. Sedentary lifestyle: a global public health problem. http://www.who.int/world-health-day/ (acessado em 10/Mar/2014). 
9. Babyac MA, Blumenthal JA, Herman S, Khatri P, Doraiswamy M, Moore K, et al. Exercise treatment for major depression: maintenance of therapeutic benefit at 10 months. Psychosom Med 2000; 62:633-8.

10. World Health Organization. Global recommendations on Physical Activity for Health. Geneva: World Health Organization; 2010.

11. Sofi F, Capalbo A, Marcucci R, Gori AM, Fedi S, Macchi C, et al. Leisure time but not occupational physical activity significantly affects cardiovascular risk factors in an adult population. Eur J Clin Invest 2007; 37:947-53.

12. Wang Y, Tuomilehto J, Jousilahti P, Antikainen R, Mähönen M, Katzmarzyk PT, et al. Occupational, commuting, and leisure-time physical activity in relation to heart failure among finnish men and women. J Am Coll Cardiol 2010; 56:1140-8.

13. Holtermann A, Marott JL, Gyntelberg F, Søgaard K, Suadicani P, Mortensen OS, et al. Occupational and leisure time physical activity: risk of all-cause mortality and myocardial infarction in the Copenhagen City Heart Study. A prospective cohort study. BMJ Open 2012; 2:e000556.

14. Peeters G, van Gellecum YR, van Uffelen JGZ, Burton NW, Brown WJ. Contribution of house and garden work to the association between physical activity and well-being in young, mid-aged and older women. Br J Sports Med 2014; 48:996-1001.

15. Medina C, Janssen I, Campos I, Barquera S. Physical inactivity prevalence and trends among Mexican adults: results from the National Health and Nutrition Survey (ENSANUT) 2006 and 2012. BMC Public Health 2013; 13:1063.

16. Knuth AG, Bacchieri G, Victora CG, Hallal PC. Changes in physical activity among Brazilian adults over a 5-year period. J Epidemiol Community Health 2010; 64:591-5.

17. Acheson D. Independent inquiry into inequalities in health. London: The Stationery Office; 1998.

18. Marmot M, Friel S, Bell R, Houweling TA, Taylor S; Commission on Social Determinants of Health. Closing the gap in a generation: health equity through action on the social determinants of health. Lancet 2008; 372:1661-9.

19. Federico B, Falese L, Marandola D, Capelli G. Socioeconomic differences in sport and physical activity among Italian adults. J Sports Sci 2013; 31:451-8.

20. Dowler E. Inequalities in diet and physical activity in Europe. Public Health Nutr 2001; 4:701-9.

21. Kleinschmidt I, Hills M, Elliott P. Smoking behaviour can be predicted by neighbourhood deprivation measures. J Epidemiol Community Health 1995; 49 Suppl 2:S72-7.

22. Chinn DJ, White M, Harland J, Drinkwater C, Raybould S. Barriers to physical activity and socioeconomic position: implications for health promotion. J Epidemiol Community Health 1999; 53:191-2
23. Liberatos P, Link BG, Kelsey JL. The measurement of social class in epidemiology. Epidemiol Rev 1988; 10:87-121.

24. Marshall SJ, Jones DA, Ainsworth BE, Reis JP, Levy SS, Macera CA. Race/Ethnicity, social class, and leisure-time physical inactivity. Med Sci Sports Exerc 2007; 39:44-51.

25. Muntner P, Gu D, Wildman RP, Chen J, Qan W, Whelton PK, et al. Prevalence of physical activity among Chinese adults: results from the International Collaborative Study of Cardiovascular Disease in Asia. Am J Public Health 2005; 95:1631-6.

26. Inquérito de Saúde no Município de São Paulo, ISA-Capital 2003. http://www.fsp.usp.br/isa-sp/ old/index_arquivos/Page3157.htm (acessado em $16 / \mathrm{Jan} / 2015)$

27. Inquérito de Saúde no Município de São Paulo, ISA-Capital 2008. http://www.fsp.usp.br/isa-sp/ old/index_arquivos/Page1494.htm (acessado em 16/Jan/2015).

28. Matsudo S, Araújo T, Matsudo V, Andrade D, Andrade E, Oliveira LC, et al. Questionário internacional de atividade física (IPAQ): estudo de validade e reprodutibilidade no Brasil. Rev Bras Ativ Fís Saúde 2001; 6:5-18.

29. IPAQ. Guidelines for Data Processing and Analysis of the International Physical Activity Questionnaire (IPAQ). http://www.ipaq.ki.se/scoring.pdf (acessado em 24/Out/2010).

30. Wilkinson R, Marmot M. Social determinants of health: the solid facts. Copenhagen: World Health Organization; 2003.

31. Trost S, Owen N, Bauman A, Sallis J, Brown W. Correlates of adults' participation in physical activity: review and update. Med Sci Sports Exerc 2002; 34:1996-2001.

32. Hamer M, Chida Y. Active commuting and cardiovascular risk: a meta-analytic review. Prev Med 2008; 46:9-13.

33. Williams PT. Dose-response relationship of physical activity to premature and total all-cause and cardiovascular disease mortality in walkers. PLoS One 2013; 8:e78777.

34. Bauman A, Ma G, Cuevas F, Omar Z, Waqanivalu T, Phongsavan P, et al. Cross-national comparisons of socioeconomic differences in the prevalence of leisure-time and occupational physical activity, and active commuting in six Asia-Pacific countries. J Epidemiol Community Health 2011; 65:35-43.

35. Knuth AG, Bacchieri G, Victora CG, Hallal PC. Changes in physical activity among Brazilian adults over a 5-year period. J Epidemiol Community Health 2010; 64:591-5.

36. Matsudo VK, Matsudo SM, Araújo TL, Andrade DR, Oliveira LC, Hallal PC. Time trends in physical activity in the state of São Paulo, Brazil: 2002-2008. Med Sci Sports Exerc 2010; 42:2231-6.

37. Hallal PC, Knuth AG, Reis RS, Rombaldi AJ, Malta DC, Iser BP, et al. Time trends of physical activity in Brazil (2006-2009). Rev Bras Epidemiol 2011; 14 Suppl 1:S53-60. 
38. Hallal PC, Matsudo SM, Matsudo VKR, Araujo TL, Andrade DR, Bertoldi AD. Physical activity in adults from two Brazilian areas: similarities and differences. Cad Saúde Pública 2005; 21:573-80.

39. Ng SW, Howard AG, Wang HJ, Su C, Zhang B. The physical activity transition among adults in China: 1991-2011. Obes Rev 2014; 15 Suppl 1:27-36.

40. Cornelio CI, García M, Schiaffino A, Borrès JM, Nieto FJ, Fernández E, et al. Changes in leisure time and occupational physical activity over 8 years: the Cornellè Health Interview Survey Follow-Up Study. J Epidemiol Community Health 2008; 62:239-44.

41. Del Duca GF, Rombaldi AJ, Knuth AG, Azevedo MR, Nahas MV, Hallal PC. Associação entre nível econômico e inatividade física em diferentes domínios. Rev Bras Ativ Fís Saúde 2009; 14:123-31.

42. Gomez LF, Duperly J, Lucumi DI, Gamez R, Venegas AS. Nivel de actividad física global en la población adulta de Bogotá (Colombia). Prevalencia y factores asociados. Gac Sanit 2005; 19:206-13.

43. He XZ, Baker DW. Differences in leisure-time, household, and work-related physical activity by race, ethnicity, and education. J Gen Intern Med $2005 ; 20: 259-66$.

44. Costa EFPVV, Florindo AA. Atividade física em diferentes domínios e sua relação com a escolaridade em adultos do distrito de Ermelino Matarazzo, zona leste de São Paulo, SP. Rev Bras Ativ Fis Saúde 2010; 15:151-6.

45. Meseguer CM, Galan I, Herruzo R, Rodriguez-Artalejo F. Trends in leisure time and occupational physical activity in the Madrid region, 1995-2008. Rev Esp Cardiol 2011; 64:21-7.

46. Jurakić $\mathrm{D}$, Pedišić $\mathrm{Z}$, Andrijašević M. Physical activity of Croatian population: cross-sectional study using international physical activity questionnaire. Croat Med J 2009; 50:165-73.

47. Talaei M, Rabiei K, Talaei Z, Amiri N, Zolfaghari B, Kabiri P, et al. Physical activity, sex, and socioeconomic status: a population based study. ARYA Atheroscler 2013; 9:51-60.

48. Mäkinen T, Borodulin K, Laatikainen T, Fogelholm M, Prättälä R. Twenty-five year socioeconomic trends in leisure-time and commuting physical activity among employed Finns. Scand J Med Sci Sports 2009; 19:188-97.

49. Salles-Costa R, Werneck GL, Lopes CS, Faerstein E. Associação entre fatores sócio-demográficos e prática de atividade física de lazer no Estudo PróSaúde. Cad Saúde Pública 2003; 19:1095-105.

50. Medina C, Janssen I, Campos I, Barquera S. Physical inactivity prevalence and trends among Mexican adults: results from the National Health and Nutrition Survey (ENSANUT) 2006 and 2012. BMC Public Health 2013; 13:1063.
51. Arango CM, Páez DC, Reis RS, Brownson RC, Parra DC. Association between the perceived environment and physical activity among adults in Latin America: a systematic review. Int J Behav Nutr Phys Act 2013; 10:122.

52. Bauman AE, Reis RS, Sallis JF, Wells JC, Loos RJ, Matin BW. Correlates of physical activity: why are some people physically active and others not? Lancet 2012; 380:258-71.

53. van Lenthe FJ, Brug J, Mackenbach JP. Neighbourhood inequalities in physical inactivity: the role of neighbourhood attractiveness, proximity to local facilities and safety in the Netherlands. Soc Sci Med 2005; 60:763-75.

54. Lima AV, Fermino RC, Oliveira MP, Añez CRR, Reis RS. Distância percebida até as instalações de lazer e sua associação com a prática de atividade física e de exercícios em adolescentes de Curitiba, Paraná, Brasil. Cad Saúde Pública 2013; 29:1507-21.

55. Martin S, Carlson S. Barriers to children walking to or from school-United States, 2004. MMWR Morb Mortal Wkly Rep 2005; 54:949-52.

56. de Rezende LF, Azeredo CM, Canella DS, Claro RM, Castro IR, Levy RB, Luiz OC. Sociodemographic and behavioral factors associated with physical activity in Brazilian adolescents. BMC Public Health 2014; $14:$ e485.

57. Fonseca Mde J, Faerstein E, Chor D, Lopes CS. Validade de peso e estatura informados e índice de massa corporal: estudo pró-saúde Rev Saúde Pública 2004; 38:392-8.

58. Benítez-Porres J, Delgado M, Ruiz JR. Comparison of physical activity estimates using International Physical Activity Questionnaire (IPAQ) and accelerometry in fibromyalgia patients: the Al-Andalus study. J Sports Sci 2013; 31:1741-52.

59. Sabia S, van Hees VT, Shipley MJ, Trenell MI, Hagger-Johnson G, Elbaz A, et al. Association between questionnaire- and accelerometer-assessed physical activity: the role of sociodemographic factors. Am J Epidemiol 2014; 179:781-90.

60. Rzewnicki R, Vanden Auweele Y, De Bourdeaudhuij I. Addressing overreporting on the International Physical Activity Questionnaire (IPAQ) telefone survey with a population sample. Public Health Nutr 2003; 6:299-305.

61. Hallal PC, Simões EJ, Reichert FF, Azevedo MR, Ramos LR, Pratt M, et al. Validity and reliability of the telephone-administered international physical activity questionarire in Brazil. J Phys Act Health 2010; 7:402-9.

62. Blair SN. Physical activity, epidemiology, public health and the American College of Sports Medicine. Med Sci Sports Exerc 2003; 35:1463.

Recebido em 29/Ago/2014

Versão final reapresentada em 06/Fev/2015

Aprovado em 02/Mar/2015 